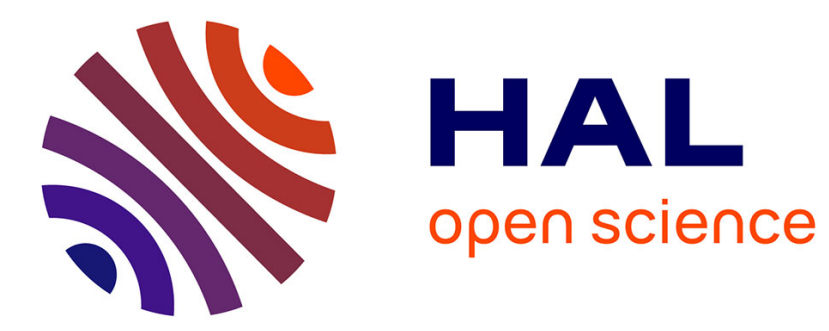

\title{
Some Aspects of Tuning and Clean Intonation in Reed Instruments
}

Jean-Pierre Dalmont, Bruno Gazengel, Joël Gilbert, Jean Kergomard

\section{To cite this version:}

Jean-Pierre Dalmont, Bruno Gazengel, Joël Gilbert, Jean Kergomard. Some Aspects of Tuning and Clean Intonation in Reed Instruments. Applied Acoustics, 1995, 46 (1), pp.19-60. hal-00475069

\section{HAL Id: hal-00475069 \\ https://hal.science/hal-00475069}

Submitted on 21 Apr 2010

HAL is a multi-disciplinary open access archive for the deposit and dissemination of scientific research documents, whether they are published or not. The documents may come from teaching and research institutions in France or abroad, or from public or private research centers.
L'archive ouverte pluridisciplinaire HAL, est destinée au dépôt et à la diffusion de documents scientifiques de niveau recherche, publiés ou non, émanant des établissements d'enseignement et de recherche français ou étrangers, des laboratoires publics ou privés. 
TITLE: Some aspects of tuning and clean intonation in reed instruments

AUTHORS: J.P. Dalmont, B. Gazengel, J. Gilbert, J. Kergomard

ADDRESS: Laboratoire d'Acoustique de l'Université du Maine, URA CNRS 1101

Avenue Olivier Messiaen, BP535

72017 Le Mans Cedex, FRANCE.

Number of pages: 43

Number of figures: 26 


\section{SUMMARY}

The influence of the first and second resonance frequencies on tuning, timbre (or tone colour) and ease of playing is investigated for reed instruments, such as the clarinet, alto saxophone and oboe. Theoretical analyses of the effects of the reed and the player

embouchure (i.e. lip position and pressure on the reed) are reviewed, as well as the consequences of inharmonicity in the resonance frequencies. This review allows us to present interesting interpretations of the numerous experiments reported here. Three kinds of results are given : i) comparison of playing frequencies and first resonance frequencies, for several fingerings with or without open register hole, leading to the definition of a frequency independent length correction for the embouchure, ii) examination of the effect of inharmonicity of the two first resonance frequencies on both tone colour and ease of playing, the causes coming from either the player embouchure or the instrument construction, iii) comparison between theory and experiment for the inharmonicity produced by the changes in conicity in oboes, leading to an interpretation of maker's choices. The results show how judicious use of simple tools, such as calculations or measurements of input impedance or playing data obtained using an artificial mouth, can be of help to the understanding of the instrument construction and the instrument designer. 


\section{Introduction.}

During the last 30 years, reed instruments have been the subject of numerous theoretical and experimental studies. These studies can be divided into two kinds : on one hand, many authors aim at describing more and more precisely one aspect of the instrument, e.g. the sound propagation in the resonator, the effect of a side hole, the mechanical behaviour of the reed, the hydrodynamics of the air entering between reed and mouthpiece. Besides that, some authors analyse the regimes of oscillation of the instrument. Obviously the significance of their results depend on the quality of the modelling of each element of an instrument. An important question is : what is the usefulness of these studies to contemporary instrument design ? Although a complete answer is beyond the scope of the present paper, but we hope to give some partial answers, not only based upon theory, but also on numerous experiments.

In the preliminary stage of the construction of an instrument, a complete and accurate prediction of its regimes of oscillation, including of the information concerning the fundamental frequencies and spectra of steady-state regimes, and the transient regimes, could be useful for the instrument maker. Nevertheless, a more accessible objective can be the prediction of the fundamental frequencies of the periodic regimes, and then of the tuning of the instrument. As is well known, the fundamental frequencies for a single reed instrument (e.g. a clarinet or a saxophone) are controlled mainly by one (or several) air column resonances. In order to modify the tuning of a fingering of a clarinet or a saxophone, it is often sufficient to correct the resonance frequency. This effect was used successfully in particular for the design of micro-interval systems for the flute and the clarinet (see Kergomard and Meynial (1988)). Nevertheless the influence of the embouchure of the instrumentalist is far from negligible, and he is actually able to correct the tuning by modifying the lip position or pressure on reed. 
In order to clarify these simple ideas and to highlight their limits, it would be interesting to start making a comparison of the fundamental frequencies of the periodic regimes (in this paper called the "playing frequencies") and the corresponding resonance frequencies for the whole range of a given instrument, for a given embouchure. Such results are rather rare in the literature : we refer to Backus (1963) for the clarinet, Nederveen (1969) for various woodwind instruments, Coltman (1968) and Lyons (1981) for flutes. The results presented in section 3 concern clarinets and alto saxophones, by using i) an artificial mouth (together with a blowing machine) for generating the playing frequencies, allowing us to maintain a constant embouchure throughout a register, ii) an input impedance measurement device for establishing the resonance frequencies. The differences in the frequencies thus obtained are converted into a length correction, $\Delta \ell$. This concept has been used by many authors (e.g. Nederveen (1969), Kergomard and Meynial (1988)) and is defined as follows : the difference between playing and resonance frequencies is regarded as an equivalent extension of the air column. It is especially useful when the length correction is independent of the frequency, i.e. of the fingering. In the presented cases, we define it as a characterisation of the embouchure effect, as explained in section 2. In this section we also summarize elementary theories of the relationship between input impedance and self-sustained oscillations.

Section 4 is devoted to the analysis of a more complicated situation, when a combination of resonance frequencies determine the playing frequency. In that case, a shift $\Delta \mathrm{f}$ of a resonance frequency does not necessarily produce the same shift in the playing frequency. Moreover, difficulties in playing can occur, as for the alto saxophone provided with micro-interval systems (Kergomard and Meynial (1988)). Bouasse (1929), and Benade (1976) (see also Fletcher (1978)), postulated that a good harmonicity of the resonance frequencies for a given fingering is essential in order to obtain satisfactory tuning, ease of playing and tone colour. Theoretical works have examined this assumption, but precise experimental measurements are rare. From the study of the influence of both the player embouchure and micro-interval systems, we found that for a small inharmonicity of the 
resonance frequencies, the tone colour is distorted ; for a larger inharmonicity, major difficulties in intonation can occur. The results are presented for the first register of the clarinet and the alto saxophone, with emphasis on certain particular fingerings.

Finally, section 5 analyses in detail one particular cause of inharmonicity : the double or triple conicity of oboes, i.e. the narrowing of the bore profile. We will prove both theoretically and experimentally that it corresponds to a maker's choice in reducing the inharmonicity of the resonances, by the truncation of the cone apex and the effects of the reed and the mouthpiece.

\section{$\underline{\text { 2. Input impedance and self-excited oscillations. }}$}

\subsection{An elementary physical model of woodwinds.}

For a description of the excitation of a woodwind instruments we prefer to use an elementary model, because of its simplicity. As a matter of fact many assumptions would require further discussion (for a more sophisticated model, see for example Hirschberg et al. (1991)).

We model the reed as a mechanical oscillator acting as a pressure-controlled valve which modulates the flow blown into the pipe of a musical instrument. The single reed of a clarinet or a saxophone is a thin piece of cane attached to the mouthpiece of the instrument (see Figure 1). The reed channel is assumed to have a rectangular cross section.

The reed is often assumed to be an oscillator with a single degree of freedom, an assumption which we adopt here (see for example Das (1931), Backus (1963), Worman (1971), Wilson and Beavers (1974)). The equation of motion of the reed is that of mass/spring system controlled by the pressure difference on both sides of it :

$$
\frac{d^{2} y}{d t^{2}}+g_{r} \frac{d y}{d t}+\omega_{r}^{2} y=\frac{-\Delta p}{\mu_{r}} \quad \text { with } \quad \Delta p=p_{m}-p
$$


where $\mathrm{y}$ is the dynamic reed displacement, $\mathrm{g}_{\mathrm{r}}$ is the reed damping factor, $\omega_{\mathrm{r}}$ is its angular eigenfrequency, $\mu_{\mathrm{r}}$ is an equivalent mass per unit area, $\mathrm{p}_{\mathrm{m}}$ is the pressure in the player's mouth, assumed to be constant, and $\mathrm{p}$ is the acoustic pressure inside the mouthpiece (see Figure 1). It must be pointed out that the term $\mu_{\mathrm{r}}$ is difficult to determine ; it is not the mass of the reed divided by its area. In most cases of woodwind applications, the inertial term can be ignored and then only the stiffness $\mu_{\mathrm{r}} \omega_{\mathrm{r}}{ }^{2}$ and the damping coefficient are needed (see Nederveen (1969)). If damping is ignored too, the alternative displacement is proportional to the acoustic pressure $\mathrm{p}$.

The acoustic pressure $\mathrm{p}$ is commonly calculated assuming a linear acoustic response of the pipe to the volume flow entering into the pipe $u_{i n}$. The linear properties of the tube resonator are described by the input impedance $Z_{\text {in }}$ or the input admittance $Y_{\text {in }}$ in the frequency domain (throughout the paper, capital characters are used for the Fourier Transform of quantities in the time domain, which are indicated by small characters) :

$$
\mathrm{P}=\mathrm{Z}_{\text {in }} \mathrm{U}_{\text {in }} \quad \text { or } \quad \mathrm{U}_{\text {in }}=\mathrm{Y}_{\text {in }} \mathrm{P}
$$

In dynamical regimes the flow entering through the reed channel $\mathrm{u}$ is divided into two parts, the flow entering into the pipe $u_{i n}$, and the flow induced by the reed movement $u_{r}$ (equation 3). We suppose that the latter is proportional to the velocity of the reed tip and also proportional to the pressure derivative in the low frequency approximation (equation 4):

$$
\begin{aligned}
& u=u_{i n}+u_{r} \\
& u_{r}=S_{r} \frac{d y}{d t}
\end{aligned}
$$

where $S_{r}$ is an effective area of the reed. 
The simplest model to calculate the flow $u$ from the pressure difference is the equation (5) derived from the stationary Bernoulli equation :

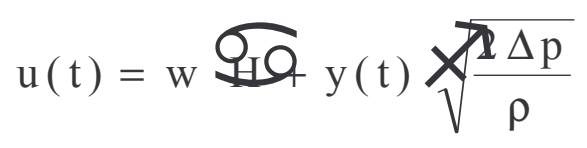

where $\rho$ is the air density, $w$ the width of the reed channel, $H$ the height of the slit in the absence of flow.

The key assumption behind the derivation of equation (5) is that the flow separates from the wall at the end of the reed channel (1mm length) and that we neglect pressure recovery by mixing of the jet with the air in the mouthpiece. A result of this flow separation is that the pressure in the reed channel is equal to the pressure in the mouthpiece if we assume a uniform reed channel cross section (see Hirschberg et al. (1990)).

In this basic model, the reed stiffness (and therefore the natural frequency) is constant during reed motion, although it can be seen from the curvature of the lay that this is certainly not the case. The system involving the above equations (1 to 5) has a solution independent of time (a singular point) for the dynamical system, corresponding to a zero pressure in the mouthpiece (for simplicity, we neglect friction losses in the tube at zero frequency), a d.c. volume velocity $\mathrm{u}_{\mathrm{O}}$, entering the mouthpiece, and an equilibrium reed position $\mathrm{y}_{\mathrm{O}}$, for the reed, as follows :

$$
\left\{\begin{array}{l}
=\frac{1}{\mu_{\mathrm{r}} \omega_{\mathrm{r}}{ }^{2}} \mathrm{p}_{\mathrm{m}} \\
=\sqrt{\frac{2 \mathrm{p}_{\mathrm{m}}}{\rho}}
\end{array}\right.
$$


In addition to the set of equations (6), a supplementary condition is required : we remark that this set of equations is valid as long as $p_{m}<\mu_{r} \omega_{r}^{2} H$ the pressure at which the reed closes the slit and volume velocity $\mathrm{u}_{\mathrm{O}}$ becomes zero.

In the following we will sometimes use a simpler, but sufficiently realistic, physical model in the modelling of the reed : only the stiffness term is retained, so that the non-linear relation relating pressure and volume velocity becomes independent of time and the knowledge of the pressure at time $t$ is sufficient for the calculation of the volume velocity. The dynamical reed volume velocity $u_{\mathrm{r}}$ is taken into account : volume velocity conservation implies that it is convenient to consider a modified resonator, with impedance $Z$, equivalent to the input impedance $Z_{i n}$ of the instrument and a reed impedance $Z_{r}$ in parallel, defined as follows :

$$
\mathrm{P}=\mathrm{Z}_{\mathrm{r}} \mathrm{U}_{\mathrm{r}} \quad \text { with } \quad \mathrm{Y}_{\mathrm{r}}=\frac{1}{\mathrm{Z}_{\mathrm{r}}}=\mathrm{jC}_{\mathrm{o}} \omega \text { and } \mathrm{C}_{\mathrm{o}}=\frac{\mathrm{S}_{\mathrm{r}}}{\mu_{\mathrm{r}} \omega_{\mathrm{r}}^{2}}
$$

then:

$$
\mathrm{Z}^{-1}=\mathrm{Z}_{\mathrm{in}}^{-1}+\mathrm{Z}_{\mathrm{r}}^{-1} \quad \text { or } \quad \mathrm{Y}=\mathrm{Y}_{\mathrm{in}}+\mathrm{Y}_{\mathrm{r}}
$$

Impedance $Z_{r}$ is capacitive, and equal to the impedance of a (fictitious) volume $\mathrm{V}$ at the input of the instrument, given by :

$$
\mathrm{V}=\rho \mathrm{c}^{2} \mathrm{C}_{0}
$$

Benade (1976) called the sum of this volume and the volume of the mouthpiece "the mouthpiece equivalent volume ". Following Nederveen (1969), in the case of a cylindrical tube one can alternatively define a length correction given by $\Delta \ell=\mathrm{V} / \mathrm{S}$, where $\mathrm{S}$ is the cross section area of the tube, i.e. in practice of the cylindrical portion of the mouthpiece. Assuming this quantity to be very small compared to the wavelength, the replacement of $Z_{\text {in }}$ by $Z$ (equation (8)) is equivalent to the extension of the instrument by a length $\Delta \ell$. 
Finally, we summarize the model by the system of two equations relating $\mathrm{p}(\mathrm{t})$ and $\mathrm{u}(\mathrm{t})$. One is non-linear and corresponds to the excitation mechanism, the other one is linear and corresponds to the passive resonator, described in the frequency domain :

$$
\begin{aligned}
& \mathrm{u}(\mathrm{t})=\mathrm{NL}[\mathrm{p} \\
& \mathrm{P}=\mathrm{Z} U
\end{aligned}
$$

Note that we clearly distinguish between the linear part of the system, i.e. the air column and the reed, and the non-linear part, i.e. the air entering into the reed channel. Therefore it is necessary to define precisely the location of the "input" of the tube for the calculation or the measurement of the input impedance $Z_{i n}$ of the instrument. The input of the resonator is for us the inlet of the mouthpiece, but, because it is not axisymmetrical in its extreme portion, we replace it by a cylindrical matching piece. The diameter of this piece is equal to that of the cylindrical part of the mouthpiece (typically $15 \mathrm{~mm}$ for a clarinet, $16 \mathrm{~mm}$ for an alto saxophone), and its volume is equal to the volume of the mouthpiece.

\subsection{Linear theory of stability and self-sustained oscillations.}

Solving the system of equations (1) to (5) yields the values involved in the stationary flow (equilibrium point), and the periodic oscillations. A linear approach for obtaining the solution to these equations is used by many authors (see Backus (1963), Nederveen (1969), Wilson and Beavers (1974), Fletcher (1979), Saneyoshi et al. (1987)). These authors look for the bifurcation point from which the equilibrium point is locally unstable, this bifurcation is

defined by a static pressure (the threshold blowing pressure), and for the fundamental frequency of the self-sustained periodic oscillation at this bifurcation (the threshold frequency). This analysis shows that (Wilson and Beavers (1974)) for slightly damped reeds, the threshold frequency closest to the natural frequency of the reed is excited at the lowest blowing pressure. In any case the threshold frequency is always smaller than the natural frequency of the reed (see Fletcher (1979)). Furthermore, the reed must be sufficiently 
damped in order to excite the lowest resonance frequency of the tube. It follows that the acoustic behaviour of a woodwind instrument depends mainly on the resonance frequencies of the tube. These resonance frequencies are defined as the frequencies where the input impedance modulus has a local maximum (or where the imaginary part of the input admittance is zero).

In practice, most of the periodic oscillations have a fundamental frequency slightly below the resonance frequency of the tube. In the previous subsection we have seen that in dynamical regimes, the flow entering through the reed channel is divided in two parts, the flow entering into the pipe, and the flow due to the reed movement. We have supposed that the latter is proportional to the velocity of the reed tip. It follows that, in the low frequency approximation, the effect is equivalent to a positive length correction, which explains the difference between the playing frequency and the resonance frequency of the tube. So most of the periodic oscillations have a playing frequency near a resonance frequency of the input impedance of the pipe in parallel with the reed impedance. Moreover we will see from experimental results (section 3) that, for a mouth-setting with a fixed blowing pressure, it is possible to associate an equivalent length correction (or a reed impedance), even if it is beyond the threshold of oscillation, to take into account the difference between the playing frequency and the resonance frequency of the tube itself. This length correction appears to be independent on tube length to a first approximation.

Schematically, the reed instrument is often regarded as a negative feedback loop system (Figure 2). One can analyse its linear stability. The study of the open-loop transfer function leads to the determination of the frequencies at which the feedback system is unstable. These frequencies can correspond to frequencies of self-oscillation of the system when the oscillating component for the input signal is zero : only the d.c. component of the input signal, i.e. the excess pressure produced by the lungs of the instrumentalist, delivers energy to the system. The open loop transfer function is the product of the transfer functions of the two elements of the loop : that of the amplifier $\mathrm{Y}_{\mathrm{g}}$ (sometimes called the generator 
admittance), and the feedback element, the element Y (see for example Elliot et al. (1982), Fletcher (1979), Saneyoski et al. (1987)).

The admittance $\mathrm{Y}_{\mathrm{g}}$ is defined by $\mathrm{Y}_{\mathrm{g}}=\mathrm{du} / \mathrm{d} \Delta \mathrm{p}$ at the equilibrium (defined by equation (6)). In order to determine it, we need to linearize the relation 5 near equilibrium :

$$
u=u_{o}+\left(\frac{\partial u}{\partial(\Delta p)}\right) \Delta p+\left(\frac{\partial u}{\partial y}\right) y=u_{o}+\left[\left(\frac{\partial u}{\partial(\Delta p)}\right)+\left(\frac{\partial u}{\partial y}\right)\left(\frac{d y}{d(\Delta p)}\right)\right] \Delta p
$$

The interesting transfer function of the feedback loop system (Figure 2) is :

$$
\frac{P}{P_{m}}=\frac{Y_{g} Z}{1+Y_{g} Z}
$$

The input quantity is the pressure in the mouth cavity, $\mathrm{p}_{\mathrm{m}}(\mathrm{t})$ (the impedance of the vocal tract being much lower than that of the instrument), we have assumed this quantity to be constant, then at non zero frequencies, it's oscillatory part $\mathrm{P}_{\mathrm{m}}$ is zero. The output quantity is the pressure at the input of the instrument, $p(t)$.

If for a constant supply pressure $\mathrm{p}_{\mathrm{m}}$, some non zero frequency components $\mathrm{P}$ of $\mathrm{p}(\mathrm{t})$ exist in the circuit, then an oscillating signal exists at the output (in practice it is limited, because of the nonlinearities of the system). The self-oscillation condition is satisfied if the denominator of the closed loop transfer function vanishes :

$$
1+Y_{\mathrm{g}} \mathrm{Z}=0
$$

Using $\mathrm{Y}=1 / \mathrm{Z}$, this implies $\mathrm{Y}+\mathrm{Y}_{\mathrm{g}}=0$, a complex equation involving the two following conditions :

$$
\begin{aligned}
& \operatorname{Re}\left(Y+Y_{g}\right)=0 \\
& \operatorname{Im}\left(Y+Y_{g}\right)=0
\end{aligned}
$$


When the conditions in (15) and (16) are satisfied, the feedback system is unstable, and of an oscillating nature, oscillating at a frequency determined by the corresponding threshold pressure $\mathrm{P}_{\mathrm{S}}$. This last quantity is the value of the supply pressure above which the equilibrium position is locally unstable. In our model, $\mathrm{P}_{\mathrm{S}}$ is slightly larger than $\left(\mathrm{H} \mu \omega_{\mathrm{r}}^{2}\right) / 3$, this value corresponds to the limit case for which no losses occur in the resonator $(\operatorname{Re}(Y)=0)$.

When these considerations are applied to the elementary model described at the end of section 2.1 (equations 10 and 11), for which $\operatorname{Im}\left(Y_{g}\right)$ is zero, equation (16) implies $\operatorname{Im}(Y)=\operatorname{Im}\left(Y_{\mathrm{r}}+Y_{\text {in }}\right)=0$ (see equation $\left.(8)\right)$, and the corresponding threshold oscillation frequency is the resonance frequency of the resonator including the reed motion correction. This analysis can be made independently in the vicinity of each resonance frequency of the resonator, and thus several different oscillation regimes are possible.

We will now discuss some important aspects of reed woodwinds oscillations, in the light of the previous, classical, theory with particular emphasis on the parameters at the disposal of the instrumentalist for the control of the sound.

We first consider the effect of the volume velocity induced by the reed movement $u_{r}$, which creates the term $\mathrm{Y}_{\mathrm{r}}$ above. The fundamental frequency $\mathrm{f}$ of a periodic regime of oscillation differs slightly from a given resonance frequency $f_{i}$, of the resonator. Their relative difference, $\Delta \mathrm{f} / \mathrm{f}_{\mathrm{i}}=\left(\mathrm{f}-\mathrm{f}_{\mathrm{i}}\right) / \mathrm{f}_{\mathrm{i}}$ can be estimated from the oscillation condition $\operatorname{Im}(\mathrm{Y})=0$. We assume the input impedance $\mathrm{Z}_{\text {in }}$ near the frequency $\mathrm{f}_{\mathrm{i}}$ to have the following form :

$$
Z_{\text {in }}=Z_{c} \frac{Z_{m}}{1+j 2 Q \frac{\Delta f}{f_{i}}}
$$

where $\mathrm{Z}_{\mathrm{m}}$ is the value of the dimensionless impedance maximum and $\mathrm{Q}$ is the quality factor of the peak. Then, from $\operatorname{Im}(Y)=0$ and from equations (7), (8) and (9), one can obtain the following result : 


$$
\frac{\Delta \mathrm{f}}{\mathrm{f}_{\mathrm{i}}}=2 \pi \mathrm{f} \mathrm{C}_{0} \frac{\mathrm{Z}_{\mathrm{c}}}{2 \mathrm{Q} / \mathrm{Z}_{\mathrm{m}}}=-2 \pi \mathrm{f} \frac{1}{2 \mathrm{Q} / \mathrm{Z}_{\mathrm{m}}} \frac{\Delta \ell}{\mathrm{c}}
$$

As a consequence, the relative difference between the playing frequency $\mathrm{f}$ and the resonance frequency $f_{i}$ is proportional to $f$. For a number of fingerings with similar resonances, i.e. with equal parameter $\mathrm{Q} / \mathrm{Z}_{\mathrm{m}}$, the coefficient of proportionality depends only on the reed impedance (i.e. its acoustic compliance $\mathrm{C}_{0}$ or its equivalent length $\Delta \ell$ ) and is independent on the fingering : it is a first characterisation of the embouchure of an instrumentalist, because the reed impedance depends in particular on the lip location on the reed. We notice that for a cylindrical tube with varying length, the parameter $\mathrm{Q} / \mathrm{Z}_{\mathrm{m}}$ is roughly independent of the fingering.

The simplicity of the previous results is mainly due to the assumption that $\operatorname{Im}\left(\mathrm{Y}_{\mathrm{g}}\right)$ is zero, i.e. to the low frequency approximation describing the reed by its stiffness alone. In this approximation, small variations of $\mathrm{p}(\mathrm{t}), \mathrm{y}(\mathrm{t}), \mathrm{u}(\mathrm{t})$ are in phase near the equilibrium position. Nevertheless, Wilson and Beavers (1974) have shown that the reed damping is essential for the control of the fundamental frequency of the oscillating regimes by the resonance frequencies of a clarinet. The lower lip of a clarinettist provides a supplementary, essential damping to the reed. This appears from a common experiment in which the entire reed is taken in the mouth and the unpleasant, high false note is observed : because of too small damping, the oscillating regime is controlled by the reed resonance, as for reed organ pipes (this regime is called " in tempo" by Bouasse (1929)).

Nederveen (1969) showed that the existence of the damping term $g_{r}$ in the reed equation (1) implies another frequency shift, $\Delta \mathrm{f} / \mathrm{f}_{\mathrm{i}}$, that is to say another length correction. Near the equilibrium position, $\mathrm{p}(\mathrm{t}), \mathrm{y}(\mathrm{t})$ and $\mathrm{u}(\mathrm{t})$ are no more exactly in phase, the oscillation condition being different from $\operatorname{Im}(\mathrm{Y})=0$. One can verify that $\operatorname{Im}\left(\mathrm{Y}_{\mathrm{g}}\right)$ is not zero and is proportional to frequency, and define a length correction $\Delta \ell_{1}$ from $C_{1}=\operatorname{Im}\left(Y_{g}\right) / \omega$, by :

$$
\Delta \ell_{1}=\rho c^{2} C_{1} / S
$$


Nederveen estimated the orders of magnitude for $\Delta \ell$ and $\Delta \ell_{1}$ to be $10 \mathrm{~mm}$ and $5 \mathrm{~mm}$, respectively, for a clarinet. A more precise estimate would necessite knowledge of the physical parameters of the reed and the hydrodynamical nonlinearity at the input, the measurement of these parameters independently of each other being very difficult. In the following, for the presentation of the experimental results (section 3), we only measure a global quantity $\Delta \ell$ characterising the embouchure of the instrumentalist, including both the effect of the volume velocity by the reed motion and the effect of the damping of the reed.

For higher notes, the inertia of the reed probably needs to be taken into account. In our analysis, this effect reduces the stiffness of the reed : the length correction becomes a growing function of $\omega / \omega_{\mathrm{r}}$. This fact could be significant for the higher notes.

The previous discussion on the linear stability of the equilibrium of the loop system exhibits one (or several) oscillation thresholds, associated to one (or several) modes, above which the equilibrium becomes locally unstable. This analysis is based upon a linear approach to the problem and cannot characterise regimes of oscillation of finite amplitude, depending on the nonlinearities of the system. When dealing with these regimes, it is necessary to use more sophisticated methods such as asymptotic methods giving the periodic solutions of steady-state regimes by iteration on the amplitudes of the harmonics (Schumacher (1978), Gilbert et al. (1989)), or the slowly varying phase method (Fletcher (1978)), or numerical methods in the time domain starting from initial conditions (Schumacher (1981), McIntyre et al. (1983), Keefe (1992)).

As an illustration an analytical study of the periodic solutions with finite amplitude is sometimes possible, in particular when the present simplified model is used (equations 10 and 11). Considering a periodic regime, with period $T=2 \pi / \omega$, the pressure and volume velocity can be written as :

$$
p(t)=\operatorname{Re}\left[\sum_{n=1}^{+\infty} p_{n} e^{j n \omega t}\right], p_{n} \in C
$$


$u(t)=\operatorname{Re}\left[\sum_{n=1}^{+\infty} u_{n} e^{j n \omega t}\right]=\operatorname{Re}\left[\sum_{n=1}^{+\infty} p_{n} Y(n \omega) e^{j n \omega t}\right], u_{n}$ et $Y \in C$

$\mathrm{u}(\mathrm{t})$ and $\mathrm{p}(\mathrm{t})$ are related by the non-linear, time independent relation (10), so that the quantity $\int u d p$ vanishes over a cycle T. Because of the orthogonality of the Fourier components, this implies the following relation:

$$
\sum_{n=1}^{\infty} \operatorname{Im}[Y(n \omega)] n\left|p_{n}\right|^{2}=0
$$

which can be rewritten as:

$$
\operatorname{Im}[Y(\omega)]+\sum_{n=2}^{\infty} \operatorname{Im}[Y(n \omega)] n\left|\frac{p_{n}}{p_{1}}\right|^{2}=0
$$

This relation (a necessary but no sufficient condition of oscillation) adapted from Boutillon (1991) for the analysis of the flattening effect of the bowed string and from Boutillon and Gibiat (1994) for analysis of saxophone, can be viewed as an extension of the oscillation condition $\operatorname{Im}[\mathrm{Y}(\omega)]=0$ at the threshold, for a non-purely sine shaped pressure. Another approach, closely related with this one, is the use of the "sum function", i.e. the average of the real part of the impedance for the frequencies n $\omega$, as explained by Pratt and Bowsher (1979) for brass instruments. For a non- sinusoidal oscillation (of finite amplitude), equation (23) shows the complex role of the higher order resonances and of the relative amplitudes of the higher harmonics in the resulting periodic regime. The oscillation frequency appears to be a compromise between the different resonance frequencies. Obviously, the simplest case where equation (23) is valid is the case for which all the resonance frequencies are harmonically related. We notice that the above mentioned postulate by Bouasse and Benade concerning the harmonicity of resonance frequencies implies this observation, though on the other hand this observation cannot considered as a proof of this postulate!

The previous results were analysed by Worman (1971) using a less restrictive model. Nevertheless this kind of analysis is implicitly based upon the existence of small, quasi- 
sinusoidal oscillations in the vicinity of the threshold, i.e. upon the existence of a direct bifurcation : the amplitude $\mathrm{p}_{1}$ is assumed to tend to zero when the supply pressure approaches the threshold pressure, so that $\left|p_{n} / p_{1}\right|$ tends to zero. This is not always the case, Grand et al. (1994) have shown that the bifurcation at the threshold of instability of the equilibrium position is not systematically direct, but can be inverse. Moreover, the height of the second resonance and the inharmonicity of its frequency with respect to the first resonance frequency are critical parameters for the kind of this bifurcation. Obviously, the nature of the nonlinearity is essential too.

\subsection{Consequences for woodwinds tuning and design.}

The prime factor in determining the quality of a woodwind instrument is its tuning. For the case of a periodic sound, the pitch perception is directly determined by its fundamental frequency. The prediction and control of the fundamental frequency (also called the playing frequency) of periodic regimes of oscillation corresponding to a given fingering is essential. In most cases, the playing frequencies are close to the resonance frequencies of the instrument, and this fact justifies the numerous studies on the measurement and the calculation of the input impedance of resonators with various geometries is justified (for example Bouasse (1929), Nederveen (1969), Backus (1975), Plitnik and Strong(1979)). Although details of the connecting conditions for multiple pipe combinations are not yet known, reasonable estimates can be made. Then straightforward calculations of these pipe combinations can be carried out. For a large number of side holes, a software package (for example RESONANS, 1984) can be used, it calculates the passive resonance frequencies for all fingerings. Within the limits of validity of this rule, it is possible to control the tuning of a wind instrument by controlling the value of the first resonance frequencies for each fingering. In order to shift the playing frequency by $\Delta \ell$, a shift in the corresponding resonance frequency of the same amount is enough. An application of this simple principle has been made for the design of micro-interval systems, translating a complete register from a given constant interval, for the flute and the clarinet (see Kergomard and Meynial (1988)). 
In section 2.2, we have shown that when using a simple model, the difference between playing frequency and resonance frequency characterises the embouchure, and is independent of the fingering. Experimentaly demonstrating this is delicate, because of the difficulty for an instrumentalist to maintain stable values for the reed parameters, independent of the fingering. We in particular refer to the experimental results of Nederveen (1969) (or Coltman (1968) for the flute), where measured playing frequencies were compared to calculated or measured resonance frequencies. To overcome this difficulty, we have constructed an artificial mouth (Gazengel, 1994).

An additional fact to be mentioned is that the playing frequency is not determined by a single resonance frequency. As an example, difficulties occurred for the design of microinterval systems for conical instruments (Kergomard and Meynial (1988)). When the second impedance peak is larger there than the first one, it can influence the oscillation sustaining process, if the first two peaks are not exactly harmonic. An extreme example, where the playing frequency does not correspond to any resonance frequency, is the so-called "pedal" notes of the trombone (Bouasse (1929)). The stability of this lowest regime is ensured by the higher order resonances, being approximate multiples of the playing frequency.

Simultaneous control of the first resonance frequencies, especially of their inharmonicity, is also important for the intonation and the self-sustaining of a note : we will give some examples in section 4.2. As noted above, the analysis of such regimes is complicated, and today this kind of analysis remains largely qualitative. The instrument maker needs to adjust the first resonance of every fingering in order to define the pitch of the first regime, but he needs to adjust the higher resonance frequencies too. There are three reasons why this is of particular importance for conical woodwinds : (i) the tone colour of the first register needs to be homogeneous and not distorted (see the critical example of the first register of the oboe in section 5) (ii) the attack of the lowest notes must to be easy (see the example of an alto saxophone in section 4.2) (iii) the register jumps need to be accurate (octave for conical woodwinds, twelfth for clarinets). Another, completely different objective 
is the search for fingerings with very inharmonic resonances in order to obtain periodic or quasi-periodic complex regimes, i.e. multiphonic tones (see Backus (1978), Castellengo (1982)).

\section{Tuning study.}

\subsection{Comparison between playing and resonance frequencies of clarinets and alto saxophones.}

In general common wind instruments like the clarinet and the alto saxophone are well tuned : the deviation of the pitch from the standard tuning tempered scale for two adjacent notes is less than 10 cents. In order to detect such small differences, the mouthpiece needs to be well controlled and the embouchure constant over a whole register. Therefore we used an artificial mouth. We measured both the playing frequencies of the first two registers of a clarinet and an alto saxophone using the artificial mouth, and the resonance frequencies of the input impedance of these instruments.

An input impedance measurement device is used for obtaining the resonance frequencies. This device includes an electrostatic transducer as a calibrated source of volume velocity described by Dalmont and Bruneau (1991) or Dalmont and Herzog (1993). In all our impedance measurements the mouthpiece of the reed instruments is replaced by a cylinder of a volume equal to the volume of the mouthpiece. An artificial mouth supplied with a blowing machine is used for obtaining realistic oscillation regimes (for a complete description, see Gazengel (1994)). This kind of device is currently used for studying reed instruments, see for example Mc Ginnis and Gallagher (1941), Backus (1961), Bak and Domler (1987), Idogawa et al. (1991) and (1993). It allow us to maintain a constant embouchure throughout each register, ensuring embouchure stability during the playing frequencies measurement, even if there are some differences with the players embouchure. The clarinet and the saxophone are transposing instruments which means that the fingered (and written) notes are different from 
the sounding ones. The notation system adopted in this paper is the same as that used by Baines (1962).

To facilitate the understanding of the results, the frequencies (on the y - axis) are represented in a tuning diagram in which the deviation in cents is plotted with respect to reference frequencies corresponding to the tempered scale, with tuning fork at $440 \mathrm{~Hz}$. The cent is the micro-interval equal to one hundredth of a tempered semitone, or a frequency ratio of $2^{1 / 1200}$. Figure $3 a$ shows the first resonance frequency and the playing frequency with artificial mouth for a given embouchure, for every fingering of the clarinet. Figure $3 \mathrm{~b}$ shows similar results for every fingering of the second register (the fingerings are identical to those of the first register, apart from the register hole being open).

One goal of these measurements is to find an answer to the following question : is a tuning diagram of resonance frequencies a measure of the instruments tuning ? We note that the local variations of the playing frequencies are similar to those of the corresponding resonance frequencies (see for example the fingerings $b-c^{\prime}-c^{\prime} \#-d^{\prime}$ for the first register, Figure 3a). This seems to give a positive answer to our question, and to justify the efforts to obtain a theoretical prediction of the resonance frequencies from the data of the geometry of an instrument.

Similar results for the two lowest registers of an alto saxophone are shown in Figures $4 \mathrm{a}$ and $4 \mathrm{~b}$. For both the clarinet and the saxophone, the playing frequencies are lower than the measured resonance frequencies. The relative deviation $\Delta \mathrm{f} / \mathrm{f}_{\mathrm{i}}$ increases with frequency. Using equation 18, we can define a quantity independent of frequency, the length correction $\Delta \ell$, representing the mouthpiece of the instrumentalist. To do this we need to do a preliminary measurement of the parameters $\mathrm{f}_{\mathrm{i}}, \mathrm{Q}$ and $\mathrm{Z}_{\mathrm{m}}$. Figures $5 \mathrm{a}$ and $5 \mathrm{~b}$ show the results, corresponding to Figures $3 \mathrm{a}$ and $3 \mathrm{~b}$, the adjustment of the artificial mouth being different for the first two registers. Actually it is very difficult to obtain a functioning of the clarinet for the two registers with the same adjustment, as in the practical experience of an instrumentalist : 
even for one register only, it is difficult to obtain the same adjustment for the whole register. For the first register, the quantity $\Delta \ell$ can be estimated to $7 \mathrm{~mm}$, with a variation $2 \mathrm{~mm}$. On the contrary, $\Delta \ell$ seems to be dependent on the fingering for the second register : perhaps this may be attributed to a reed inertia effect (see section 2.2) ?

For an alto saxophone similar results are shown in Figures $6 \mathrm{a}$ and $6 \mathrm{~b}$, corresponding to $4 \mathrm{a}$ and $4 \mathrm{~b}$. It was extremely difficult for us to sound the lowest notes without overblowing, in accordance with the experience of beginners, so the first seven notes were left out. The choice of the material mimicking the lower lip of the saxophonist is very critical. For the first register, $\Delta \ell$ is estimated to be $7.5 \mathrm{~mm}( \pm 1.5 \mathrm{~mm})$ for a cylinder of diameter $16 \mathrm{~mm}$. For the second register, using two different adjustments of the mouth, we found respectively $6 \mathrm{~mm}$ $\pm 1 \mathrm{~mm})$ and $5 \mathrm{~mm}( \pm 0.5 \mathrm{~mm})$ : the second one is slightly tighter than the first one. For the highest notes of the second register, we again find an increase of $\Delta \ell$, especially for the first adjustment, although it is less marked than for the clarinet.

We can conclude that the characterisation of a mouthpiece to explain the discrepancy between playing and resonance frequencies, by a length correction $\Delta \ell$ is rather satisfying. Independently of our theoretical interpretation based upon a simple model for small amplitude oscillations, we can deduce that it is possible to predict the playing frequencies of a reed instrument from the measurement of the impedance of the resonator alone, with an extension of the mouthpiece of between 5 and $10 \mathrm{~mm}$. This correction can be viewed as a fitting parameter obtained from a given reference. More rigorously, it would be better to take into account a second correction for the higher notes of each register for the effect of the temperature gradient, which is non negligible at the beginning of the resonator when a musician is playing.

\subsection{Register hole effects.}

A register hole is a side chimney, with small diameter $\mathrm{d}$ but large height $\mathrm{h}$ (typically 3 and $10 \mathrm{~mm}$, respectively, for a clarinet). Its role is to make it easier to achieve the higher 
order regimes, i.e. the notes of the second register, for which the fundamental frequency is close to the second resonance frequency. Actually the register hole causes a non negligible frequency shift to the second resonance frequency. Deviations can be theoretically predicted from an elementary model (see e.g. Kergomard and Meynial (1988)) :

$$
\frac{\Delta \mathrm{f}}{\mathrm{f}}=\frac{\ell \mathrm{s}}{\mathrm{Sh}^{\prime}} \frac{\cos ^{2} \mathrm{k} \ell}{\mathrm{d} \ell}
$$

where $\ell$ is the distance of the hole from the clarinet reed tip, $\mathrm{k}=\omega / \mathrm{c}$ is the wavenumber of a plane wave, $\mathrm{s}$ and $\mathrm{S}$ are the cross section areas of the hole and the tube, respectively, $h^{\prime}=h+0.7 d$. For a saxophone, the cosine needs to be replaced by the sine.

Experimental results (see Figure 7) confirm the dependence of the deviation on the location of the hole for a clarinet : as it is well known, it is zero near the pressure node located at one third the length of a cylindrical tube. For the highest notes of the first register (from g' to $\left.\mathrm{a}^{\prime} \#\right)$, the deviation is very large. However these fingerings with open register hole are not normally used for obtaining the corresponding twelfths (from d"' to $\mathrm{f}^{\prime \prime}$ ). Instead specific fingerings employed, the oscillations occurring near the third resonance frequencies, as shown for example by Figure 8 : the input impedance curves are plotted for two fingerings of the same note $\mathrm{c}^{\prime \prime} \#$. One is the fingering of $\mathrm{f}^{\prime} \#$ of the first register with open register hole (Figure 8b), the note being too high, the other is the commonly used fingering using the third resonance (Figure 8a).

Figure 9 shows experimental results for the alto saxophone of the second resonance frequency due to the two register holes : the first one is used for the notes $d "$ to $g " \#$, the second one for the notes a" to c'"\# using the same fingerings as the first register. A single key controls of these two holes, the change in register hole being automatic between g"\# and a". As appears from Figure 9, the location seems to be an optimum for the $\mathrm{f}^{\prime \prime}$ fingering and the c'"\# fingering, respectively. 


\section{$\underline{\text { 4. Effect of resonance inharmonicity on tone colour and ease of playing. }}$}

\subsection{Results for clarinets.}

Inharmonicity of the first resonance frequencies is important for the tone colour of a wind instrument. In particular, we will show that the spectrum of the steady-state of a given note strongly depends on inharmonicity. Consider a periodic oscillation of frequency $\mathrm{f}$, close to the first resonance frequency. The amplitudes of the harmonics $p_{n}$ and $u_{n}$ of pressure and volume velocity, respectively, are related by : $p_{n}=|Z(n f)| u_{n}$. For a given value of $u_{n}, p_{n}$ is very sensitive to $Z(n f)$; it is a maximum if the $n$ 'th resonance frequency $f_{n}$ is close to $n f_{1}$, i.e. $f_{n}$ is a harmonic of $f_{1}$. Vice versa, an increasing deviation of $f_{n}$ from $\mathrm{nf}_{1}$ produces a decrease of $\mathrm{p}_{\mathrm{n}}$ and then a change in tone colour for the considered note. We will present an experimental verification of this for an alto saxophone (section 4.2). These elementary considerations are supported by our discussion in section 2 (see equation 23) or, better, by a more complete treatment (see e.g. Worman (1971)).

Some fingerings with a special tone colour are especially suitable for the analysis of the inharmonicity of the fingerings of a given register. Actually, when the first register is played with the artificial mouth, using a descending chromatic scale, some notes with special tone colour are easily detected, especially b', f '\# and a'\#, the highest note of the register. For $\mathrm{a}^{\prime} \#$, the deviation from the other spectra is very strong and evident. Figure $10 \mathrm{~b}$ shows that these notes have particular values for the inharmonicity, noted I, defined for the first register as follows : $I=f_{2} / 3 f_{1}$, where $f_{1}$ and $f_{2}$ are the first two resonance frequencies. The inharmonicity I is close to unity and can be represented in cents. It can be deduced directly by subtraction from the curves of the two first resonance frequencies (see Figure 10a).

Some notes can be obtained by using several, different fingerings. The choice between them depends on technical or aesthetical requirements. For a given note, we call "usual" the most common fingering, normally represented in the Figures, the other ones being called "unusual". The experimental values corresponding to the unusual fingerings b', f'\# and a'\# are 
plotted on the Figures 10a and 10b. The characteristics of an unusual fingering differ from those of an usual one by both tuning and tone colour. As examples, the pitch of the fork fingering for $b^{\prime}$ is lower than this of usual fingering, and, contrary to the usual fingering, the unusual fingering for $\mathrm{a}^{\prime} \#$ has a tone colour similar to this of the adjacent notes $\mathrm{g}^{\prime}$ and $\mathrm{a}^{\prime}:$ the correlation can be made with identical values for the inharmonicity of these notes.

Finally, we wanted to verify if the experimental arrangements are sufficiently accurate to detect significant differences between clarinets of different models. When comparing two clarinets, we actually found great similarities. However, the experimental results (see Figure 11) for the inharmonicity of the lower and the higher parts of the first register are somewhat different, exhibiting different compromises chosen by the makers concerning the higher part of the register. Apparently, maker A choose to accept a positive inharmonicity for the fingerings $g^{\prime}, g^{\prime} \#$, and $a^{\prime}$ in order to restrict the important (negative) inharmonicity, due to the use of the register hole, for the fingering a'\#. The tone colour of this higher part of the first register is found to be more homogeneous than that of clarinet $\mathrm{B}$. We notice that the differences in harmonicity between the two instruments are correlated to the bore profile of the upper part of the instrument (barrel and upper joint).

\subsection{Results for alto saxophones.}

\section{i) Inharmonicity and tone colour.}

We first consider the case where the amplitude of the second resonance is lower than the first one. The experiments concern the last note of the first register, fingered $c^{\prime} \#$, with possible modifications of the air column. Here, the resonator has essentially two resonances of which the second one is $5 \mathrm{~dB}$ lower than the first ( see Figure 12).

The saxophone can be modified by addition of closed side tubes on the neck (see Figure 13). In the side tubes, pistons can be moved to modify their volume. By adjusting the volumes of the closed tubes 1 and 2 we are able to shift the resonance frequencies and thereby 
to control the inharmonicity. We can see in Figure 15 that, because of its location, an increase of volume 2 causes a decrease of the first resonance frequency $f_{1}$ of $c " \#$, and no change in the second resonance frequency $\mathrm{f}_{2}$, so that the inharmonicity is increased. On the contrary, an increase of volume 1 causes decreases both $f_{1}$ and $f_{2}$ with about the same quantity (Figure 14), without variation of the inharmonicity.

We have measured that when either volume 1 or 2 is increased, the decrease of the fundamental frequency of the periodic oscillation is about-equal to the decrease of the first resonance frequency $f_{1}$. So the modification of the inharmonicity has little effect on the fundamental frequency of the oscillation, but it has great effects on the tone colour. The increase of volume 2 (case of increasing inharmonicity) damages the tone colour. On the contrary, increase of volume 1 (case of constant inharmonicity) does not change the tone colour. It is difficult to define the damage on tone colour rigorously, but we can see in Figure 16 that it is correlated to the decrease of the amplitude of the harmonic 2.

\section{ii) Inharmonicity and oscillation.}

In the previous case, the main effect of the increase of inharmonicity is on the tone colour. In other cases, however if the second resonance is at least of the same order of magnitude as the first resonance, an increase of inharmonicity can cause difficulties in the attack of the corresponding note, and it is of great importance for the player.

An example is the fingered g' of an alto saxophone (first register) : the resonator has essentially two main resonances, $f_{1}$ and $f_{2}$ (see Figure 17 ), with nearly the same amplitude. We can also observe a third resonance, $f_{3}$, but we assume that it has no influence on the oscillations because of its comparatively low amplitude.

As in the previous section, by adjusting the volumes of the closed tubes 1 and 2, we are able to shift the resonance frequencies to control the inharmonicity. We can see on Figure 14 that an increase of volume 2 causes a decrease of the first resonance frequency $\mathrm{f}_{1}$, and no 
change of the second resonance $f_{2}$, because the connection of the closed tube 1 is located at a pressure node. Then only the first resonance is shifted, and the inharmonicity increases. The consequences are the following : an increase of the volume 2 causes a decrease of the pitch of $\mathrm{g}^{\prime}$. But it cannot be decreased indefinitely. For a particular value of the volume, the oscillation becomes quasi-periodic with a beat frequency of $\mathrm{f}_{2}$ minus two times $\mathrm{f}_{1}$. Up to this change in behaviour, it appears from measurements that the decrease of the fundamental frequency of the periodic oscillation was less than the decrease of the first resonance frequency $\mathrm{f}_{1}$.

We have analysed the acoustic pressure signal in the case of this quasi-periodic oscillation, obtained with the fingered g', with volume 2 at its maximum and volume 1 at zero. The corresponding spectrum is a discrete spectrum, and every frequency can be regarded as a linear combination of two frequencies $f_{1}$ and $f_{2}$. Note that the discrete frequencies have been determined assuming a quasi-periodic signal, with a deconvolution around every peak by a cardinal sinusoid (the function $\mathrm{x}^{-1} \sin (\mathrm{x})$, the Fourier transform of the rectangular window used during the digital conversion).

The measured frequencies $f_{1}$ and $f_{2}$ in this discrete spectrum were verified to be the resonance frequencies of the pipe impedance in parallel with the reed impedance. To this aim we measured the resonance frequencies of the pipe in the above-described configuration and estimated the equivalent length correction of the reed impedance corresponding to the mouthsetting used during the experiment. The length correction is estimated by comparing the measured resonance frequencies with the fundamental frequencies of the corresponding periodic oscillations for other fingerings : the two fingerings used are c"\# (last note of the first register) and g" of the second register. Assuming that the equivalent volume V of a mouthsetting is independent of the fingering, we have found $\mathrm{V}=\mathrm{S} \Delta \ell$ where $\Delta \ell=10 \mathrm{~mm}$. The new resonance curve then is calculated from the measured pipe impedance with the reed impedance (characterised by a length correction of $10 \mathrm{~mm}$ ) in parallel. The new resonance frequencies are close to those measured. We can conclude that, below bifurcation, the decrease of the fundamental frequency of the periodic oscillation is less than the decrease of 
the first resonance frequency. Above the bifurcation, it seems that the frequencies of the quasi-periodic oscillations are linear combinations of frequencies which are the resonance frequencies of the pipe in parallel with the reed.

The location of the closed tube 1 is calculated in order to keep intact the inharmonicity. An increase of volume 1 causes decreases for $f_{1}$ and $f_{2}$, but no changes in the inharmonicity value (Figure 15). The effects on the oscillations are the following : an increase of volume 1 decreases the pitch of $\mathrm{g}^{\prime}$ without significant change in sound quality. We measured that the decrease of the fundamental frequency of the periodic oscillation is equal to the decrease of the first resonance frequency $f_{1}$. Contrary to the previous experiment, the oscillation is always periodic because the inharmonicity has a constant value during the modification of the resonance frequencies.

\section{iii) Mouth-setting influence on the ease of playing.}

The previous experiments were made with a constant mouth-setting (i.e. a constant reed impedance), and a variable resonator by changing the volumes of tubes 1 and 2 (variable input impedance of the tube). But a mouth modification can influence the oscillation as well. The alto saxophone bore can be represented by a conical part (the body of the instrument) completed by a volume represented by a cylindrical part (the mouthpiece). It is possible to calculate the influence of this volume on the inharmonicity of the resonance frequencies : it appears that the inharmonicity decreases with increasing volume. If the oscillation is a quasiperiodic one during the attack, a decrease of the saxophonist lip pressure on the reed (corresponding to an increase of the equivalent volume) causes a decrease of the inharmonicity sufficient to produce a periodic oscillation. Moreover, the great deviation of the inharmonicity introduced by connecting tube 2 to the pipe can be compensated for by a mouth modification by the player : actually, a variation of inharmonicity due either to a resonator modification or to a lip pressure modification has an equivalent effect on the oscillation. We have found previously that the beat frequency of the quasi-periodic oscillation is equal to $f_{2}$ 
minus two times $f_{1}$, so the saxophonist can control this beat frequency with the control of his lip-pressure on the reed. We remark that the quantitative influence of the mouth on the inharmonicity depends on the bore ; in fact there are no such effects with a cylindrical bore : a length correction in the input of the bore always implies changes in the resonance frequencies and changes in their relative inharmonicity if the bore is conical, but no changes in their relative inharmonicity if the bore is cylindrical.

Since in practice the saxophone player actually feels that the ease of playing worsens if the inharmonicity increases, we conclude that the harmonicity of resonance frequencies is linked with the ease of playing. A quasi-periodic oscillation is the limiting case of an unplayable note. The above-described experiments are an illustration of the relation between good harmonicity and ease of playing, and an experimental confirmation of earlier statements by Bouasse (1929) and Benade (1976). Three sound examples illustrating the experiments of this section 4.2 are available on a demonstrative CD (Dalmont and Gilbert (1993)).

\section{Effect of changes in conicity of the main bore on the inharmonicity in conical woodwinds.}

In this section we study the effect of small deviations in conicity of conical reed instruments and especially the oboe. We show theoretically that even a very small narrowing of the bore (with respect to a true cone) at the top of conical woodwind can lower the inharmonicity of the resonance frequencies. Measurements on different oboes show that this property is used by the oboe makers.

\subsection{Theoretical considerations.}

\subsubsection{Principle and hypothesis of the calculations.}

Oboes have a conical bore. Measurements on different oboes (see Nederveen (1967), and subsection 5.2 below) show that, except for the bell, the bore is not very different from a 
true cone. Therefore the resonator part of an oboe is often modelled by a truncated cone completed by a volume representing the double reed (for a discussion about this term see section 2-2). The purpose of this section is to point out the limitations of this model and particularly to show the relation between the inharmonicity of the resonance frequencies and the actual geometry of the bore.

Neglecting dissipation losses, the resonance frequencies of a resonator tube can be calculated, by setting $\mathrm{Y}=0$ where $\mathrm{Y}$ is the input admittance of the resonator. For a complete cone (no truncation) of length $\mathrm{L}$, open at the end, this leads to the equation : $\tan \mathrm{kL}=0$ where $\mathrm{k}=\omega / \mathrm{c}$ is the wavenumber (see e.g. Ayers et al. (1985)). The imaginary part of the radiation impedance is incorporated in the tube length by a correction on the length of the cone. In that ideal case resonances are exactly harmonic.

For other horns, deviating not too much from a complete cone, the equation $\mathrm{Y}=0$ can be written as :

$$
\tan \mathrm{k}(\mathrm{L}+\Delta \ell)=0
$$

where $\mathrm{L}$ is the length of the complete cone, and $\Delta \ell$ a length correction depending on the bore deviation of the complete cone, and also on the frequency (see for a general discussion Kergomard and Meynial (1988)). In that case resonances are exactly harmonic only if $\Delta \ell$ is independent on frequency. If the deviations are located near the top of the instrument, the harmonicity is ensured for every fingering, i.e. for every equivalent length of the cone, so long as the toneholes do not produce inharmonicity.

A series expansion of $\Delta \ell$ versus frequency can be done as follows :

$$
\Delta \ell(\mathrm{kx})=\Delta \ell_{\mathrm{o}}+\sum_{\mathrm{n}=1}^{\mathrm{n}=\infty} \mathrm{a}_{\mathrm{n}} \underset{\mathrm{S \alpha} X}{ }
$$


where $\mathrm{x}$ is a length. $\Delta \ell_{\mathrm{O}}$ is the first term of the series expansion and is a frequency independent length and $a_{n}$ are constants (with the dimension of length). Resonance frequencies are the more harmonic the smaller $\left(\Delta \ell-\Delta \ell_{0}\right)$ is. A way to minimise the harmonicity quantity is then to cancel the first successive terms in the development of $\left(\Delta \ell-\Delta \ell_{0}\right)$. Then equation (21) can be rewritten in the following form :

$$
\tan \mathrm{kL}^{\prime}=\varepsilon \quad \text { with } \mathrm{L}^{\prime}=\mathrm{L}+\Delta \ell_{\mathrm{o}} \quad \text { and } \quad \varepsilon=-\tan \mathrm{k}\left(\Delta \ell-\Delta \ell_{\mathrm{o}}\right)
$$

It is equivalent to cancel the first successive terms of the expansion in $\varepsilon \mathcal{Y}$ or in $\left(\Delta \ell-\Delta \ell_{\mathrm{o}}\right)$ (we notice that $\varepsilon$ is at least in the third order in $\mathrm{kx}$ ). The inharmonicity I between the first two resonance frequencies can be deduced from $\varepsilon \nsucceq$ by the following relation : $\mathrm{I}=\frac{1+\mathrm{arctan}}{1+\arctan }-1$ where $\mathrm{k}_{1}$ and $\mathrm{k}_{2}$ are the first two solutions of the equation $(21)$.

These wave numbers, $\mathrm{k}_{1}$ and $\mathrm{k}_{2}$, can be easily calculated by an iterative process, the first estimated values of $k_{1}$ and $k_{2}$ being $k_{1}^{(1)}=\pi / L^{\prime}$ and $k_{2}^{(1)}=2 k_{1}^{(1)}$. The following values of $\mathrm{k}_{1}^{(\mathrm{n})}$ and $\mathrm{k}_{2}^{(\mathrm{n})}$ are deduced from the relation $\tan \mathrm{k}_{\mathrm{i}}^{(\mathrm{n})}=\varepsilon \mathbf{W}\left(\mathbf{W}^{-1)^{\prime}}\right.$, with $\mathrm{i}=1,2$. In practice $\mathrm{k}_{\mathrm{i}}^{(2)}$ is a sufficiently good approximation for the calculation of I.

We finally note that identification of series expansions or continued fraction expansions is equivalent, therefore we know that the validity can be largely extended to frequencies for which the wavelength can be shorter than the length x (see Kergomard (1988), especially the appendix).

\subsubsection{The case of the truncated cone.}

For a truncated cone, it is well known (see Nederveen (1969)) that to the first order in $\mathrm{kx}, \Delta \ell_{0}$ is found to be the distance between the apex of the cone to the input of the truncated cone (see Figure 18). In practice the truncated cone (the instrument) is completed 
by a volume, $\mathrm{V}$, equivalent to the embouchure, as explained in the previous sections. The condition for having $\varepsilon$ accurate to the fifth order in $\mathrm{kx}_{0}$, where $\mathrm{x}_{0}=\mathrm{V} / \mathrm{S}_{0}$ and $\mathrm{S}_{0}$ is the input cross section, is $\mathrm{x}_{0}=\ell / 3$ or $\mathrm{V}=\mathrm{S}_{0} \ell / 3$, which is the volume of the missing apex of the cone. This can be related to the statement given by Benade (1976) (see also Nederveen (1969), Benade (1974) and (1983), and Kergomard (1988) : " for a conical woodwind instrument to work properly, the equivalent volume of the reed [...] must closely match the volume of the missing part of the cone". The expression "work properly" is to be understand as a minimal inharmonicity of the resonance frequencies.

\subsubsection{The case of two successive cones}

We consider a resonator made of two successive cones of length $\mathrm{L}_{1}$ (first cone) and $\mathrm{L}_{2}$ (main cone) with a volume $\mathrm{V}$ at the input (see Figure 19). The conicity of the first cone is supposed to be slightly different of the conicity of the main cone and is characterised by the parameter $\lambda=\frac{\tan \theta_{1}}{\tan \theta}-1$ where $\theta$ and $\theta_{1}$ are the angles at the apex of respectively the main cone and the input cone.

In that case it can be shown that resonance condition can be written : $\tan \mathrm{kL}^{\prime}=\varepsilon$, where $\mathrm{L}^{\prime}=\mathrm{L}+\ell$ and

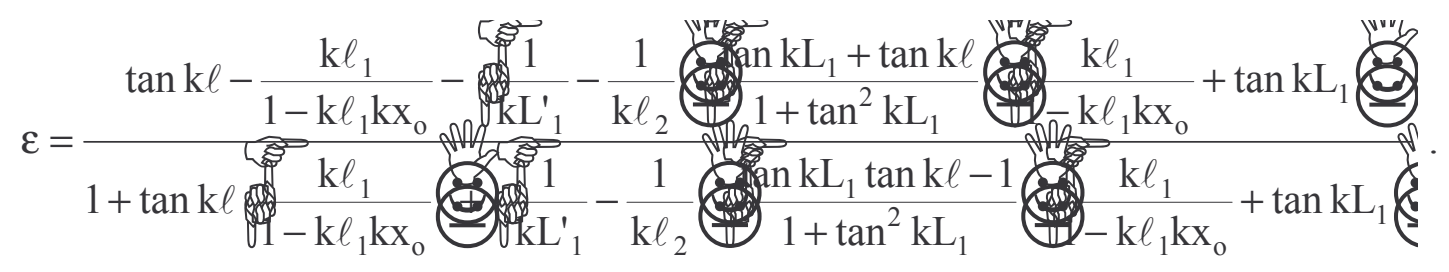

$\varepsilon$ is of the third order in $\mathrm{kL}_{1}$ and $\mathrm{k} \ell$. Notations are given on Figure 19.

This coefficient of third order term of $\varepsilon$ becomes zero when the input volume is equal to $\mathrm{V}=\frac{1}{3} \mathrm{~S}_{\mathrm{o}}+\frac{\lambda \mathrm{L}_{1}^{\prime 3}}{\ell_{1}^{2}}$ where $\mathrm{S}_{\mathrm{o}}$ is the input section of the first cone, $\ell_{1}$ is the distance from the input of the first cone to its apex and $\mathrm{L}^{\prime}{ }_{1}=\mathrm{L}_{1}+\ell_{1}$ (notations are given on Figure 19). This volume is equal to the volume difference between the complete cone of length L' and the 
system with two conicities (see Figure 20), according to the general result given by Kergomard (1988). For $\lambda=0$, we verify the result stated in the previous section.

It can be shown that for a given length $\mathrm{L}_{1}$ there is an optimum value of the parameter $\lambda$ for which the coefficient of the fifth order of $\varepsilon$ vanishes too. This optimum value of $\lambda$ is solution of the equation of the third order :

$$
\alpha^{6} \lambda^{3}-3(\alpha+2) \alpha^{5} \lambda^{2}-\alpha\left(1+5 \alpha+15 \alpha^{2}+10 \alpha^{3}+2 \alpha^{4}\right) \lambda+1=0
$$

where $\alpha=\mathrm{L}_{1} / \ell$. If we assume $\lambda<<1$ or $\alpha<<1$, this equation can be simplified into :

$$
\lambda^{-1}=\alpha\left(1+5 \alpha+15 \alpha^{2}+10 \alpha^{3}+2 \alpha^{4}\right)
$$

For example, if $\mathrm{L}_{1}=\ell(\alpha=1)$ the parameter $\lambda=0.03$ which implies a very wide change in conicity. The inharmonicity is then reduced as compared to the case of a single cone with optimum input volume as shown on Figure 21. It can also be shown that the inharmonicity is as weak as the length $\mathrm{L}_{1}$ is small but consequently the change in conicity is more important when this length is short. For example if $\mathrm{L}_{1}=\ell / 3 \quad(\alpha=1 / 3)$, then $\lambda=0.63$ which is a wide change on conicity. For the oboe, the length $\ell / 3$ is not very different from the length of the staple.

\subsubsection{Discussion.}

The previous calculations do not take into account the geometry of the input volume (including the reed volume and the effect of the reed parameters), dissipation, radiation at the end and the existence of tone holes. When all these parameters are taken into account, the optimum values of the input volume and of the relative conicity of the two cones could be different from the calculated ones.

However, the previous calculations were not intended to find an hypothetical ideal bore for the oboe, but only to show how modifications of the conicity at the input of conical 
woodwinds can affect the inharmonicity of the resonance frequencies. The exact mathematical optimum may not be the choice of an oboe maker. Firstly, the equivalent length $L^{\prime}$ of the oboe is limited $\left(0.35<L^{\prime}<0.75 \mathrm{~m}\right)$ and secondly a more important inharmonicity for greater lengths can be accepted if it implies a lower inharmonicity for small lengths. For example, Figure 22 shows the inharmonicity obtained for a non optimum conicity ratio. It shows that the theoretical optimum implies a globally lower inharmonicity but for lengths lower than $0.5 \mathrm{~m}$ the compromise with $\lambda=1.0$ is better. Another limitation of our model is that the "oboe maker" will not be limited to only two cones for the bore. The following section will explain this further.

Nevertheless the following results can be stated :

i) In all cases the inharmonicity increases when the resonating length of the tube is lower (for a fixed value of the truncation).

ii) A weak change in conicity at the top has a significant influence on the inharmonicity. An increased conicity top tends to reduce the inharmonicity.

iii) To optimise the inharmonicity the change of the top conicity will be just as important since this change occurs in a short length.

\subsection{Experiments on oboes.}

\subsubsection{Main bore measurements.}

The main bore of three different French (Rigoutat, Cabart) oboes have been measured with gages (Mitutoyo series 154 and 155) and with a calliper. The accuracy of these (very delicate) measurements is estimated to be $\pm 0.05 \mathrm{~mm}$ on the diameter and $\pm 1 \mathrm{~mm}$ on the position. The section of the bore is assumed to be circular which is not always exactly the case. The results obtained are presented on Figures 23 and 24. The Figure 24 shows the 
deviation of what Nederveen (1967) calls the "Russell standard cone", Russel (1953), i.e. the quantity $\Delta \mathrm{d}=\mathrm{d} \cup \mathbf{x}$ / 40 where $\mathrm{d}(\mathrm{x})$ is the distance to the apex of the standard cone, which is $160 \mathrm{~mm}$ from the top of the oboe.

From these curves it can be seen that the deviations from the "Russell standard cone" are very small : they are, except for the bell, less than $0.4 \mathrm{~mm}$. This bore can be schematised by successive parts of cones. Typically, excluding the bell, three cones can be distinguished (see also Nederveen (1967)) : i) the first one for the top of the oboe - with conicity significantly larger than the conicity of the "Russell standard cone" -, ii) the second one for the main part of the upper part - with conicity approximately equal to the conicity of the "Russell standard cone" -; the third one for the lower part - conicity smaller or equal to the conicity of the "Russell standard cone". We may also consider the staple of the reed which can be roughly considered as an other cone with a larger conicity (see Nederveen (1967) and Benade and Richards (1983)). Furthermore some bore discontinuities can occur at the junctions between the different parts of the oboe.

It appears that the bore is different from the model considered in section 5.1.3. but the choice of an increasing conicity can be observed three times showing that this is used by the oboe makers to optimise the tuning. Note that, for the staple, approximated by a cone of length $47 \mathrm{~mm}$ and input output sections of respectively $2.3 \mathrm{~mm}$ and $4.8 \mathrm{~mm}$, we have a $\lambda=1.2$ . This value is very similar to the non optimum value considered in Figure 22 (lower curve) showing that the practical optimum should be different from the theoretical one.

\subsubsection{Calculation and measurements of the inharmonicity of the first two resonance frequencies.}

Input impedances of virtual oboes schematised according to the drawn lines in Figure 24 have been calculated with the RESONANS program (1984). No holes were modelled but the total length of the oboe was changed by "cutting" the lower part of the oboe. The reed was modelled by its equivalent volume (cylinder of length $2.73 \mathrm{~mm}$ and diameter $12 \mathrm{~mm}$ as in 
impedance measurements) and the staple by a single cone (input radius $2.3 \mathrm{~mm}$, output radius $4.8 \mathrm{~mm}$ and length $47 \mathrm{~mm})$. Inharmonicity between the first two resonance frequencies are calculated and plotted in Figure 25b. An increase of the inharmonicity for the small lengths (higher notes) similar to the one observed on Figure 25a can be seen. The differences between the respective curves for the three oboes is significant though the differences between the bores are very small. Here we may have a clue why the oboe is a such "delicate" instrument and why oboes with approximately identical shapes can sound very differently.

Input impedance measurements were made for the three oboes considered. The input volume was taken identical for the three oboes, and chosen such as to obtain a first resonance frequency for the staple alone of $955 \mathrm{~Hz}$. This frequency was determined by blowing the reed and staple alone (see Benade (1976)). Temperature was $20^{\circ} \mathrm{C} \pm 1^{\circ} \mathrm{C}$ for all experiments.

The tuning of the three oboes is very similar as can be seen in Figure 26. In this figure the deviation of the pitch of the first resonance frequencies from equal temperament (with $\mathrm{A}=440 \mathrm{~Hz}$ ) is plotted. The difference in tuning between oboes does not exceed 20 cents (the absolute uncertainty on the measurements being estimated to be \pm 5 cents).

From the input impedance measurements the inharmonicity between the first two resonance frequencies has been calculated and plotted versus the equivalent length $\mathrm{L}$ $(\mathrm{L}=\mathrm{c} / 2 \mathrm{f})$ in Figure 25a. The curves obtained experimentally are close to the ones obtained with the RESONANS program (Figure 25.b) despite the fact that our data do not take into account the perturbation due to the closed holes according to Nederveen (1969) the closed holes can lower the frequency up to 50 cents ! In particular we can observe, on both experimental and theoretical curves, that the inharmonicity for the upper notes of the oboe B is greater than for the oboe $\mathrm{C}$ and whereas it is less for the lower notes. The inharmonicity measured for oboe A is lower than theoretically predicted.

The bore of modern oboes is not a straight cone. Many deviations from the standard cone can be observed. This is explained theoretically by the fact that the straight cone is not 
an optimum from the point of view of the inharmonicity. In practice the problem of the inharmonicity is crucial for the highest note of the first register ( $\left.c^{\prime}\right)$ where tone colour can differ much from other notes. Inharmonicity should not exceed a certain limit for these notes. To prevent this problem various compromises can be found and give good results : this allow for the oboe makers to build different patterns of their instruments.

\section{Conclusion.}

An important goal of research into the acoustics of musical instruments is to improve and facilitate building an instrument. The major difficulty lies in the achievement of instrument characterisation, precise enough for musical purpose, but simple enough to be useful for design purposes. The simple, classical assumption that the playing frequencies are equal to the first resonance frequencies sometimes give useful results, in particular for the implementation of small modifications. Nevertheless, simple corrections such as the embouchure correction, depending mainly on the player, or the register hole effect, depending on the maker, can be interesting to take into account. The present paper aims to give some experimental results concerning these effects. The existence of several resonances can be very important, especially when they are of similar magnitude and their frequencies are slightly inharmonic. The paper gives some results concerning both the effects and the sources of this inharmonicity, in the context of rather high pitched conical woodwinds such alto saxophones or oboes. The importance of the existence of several resonances may be even more important for bass instruments. Through these examples, we have demonstrated qualitatively on these examples, some phenomena. That, in general, are assumed to exist intuitively, such as the influence of inharmonicity on tone colour and ease of playing. The examples are again chosen related to the action of both the player and the maker.

Finally, we hope that by these results, other investigators and makers are stimulated to use simple tools which have became available in the last decade such as measurements or calculation of input impedances, and artificial mouth. 


\section{Acknowledgements}

We wish to thank P. Dupire, F. Giroud, F. Durand, P. Herzog and J.C. Valière for their contributions to this research project. We wish also thank N. Amir, A. Hirschberg and F. Laloé for fruitful discussions. Special thanks are due to K. Nederveen for his helpful suggestions. 


\section{References}

AYERS R.D., ELIASON L.J. and MAHGEREFTEH D. (1985) "The conical bore in musical acoustics", Am. J. Phys., 53, 528-537.

BACKUS J. (1961) "Vibrations of the reed and air column in the clarinet", J. Acoust. Soc. Am., 33, 806-809.

BACKUS J. (1963) "Small-vibration theory of the clarinet", J. Acoust. Soc. Am., 35, 305313.

BACKUS J. (1978) "Multiphonic tones in the woodwind instruments", J. Acoust. Soc. Am., 63, 591-599.

BAINES A. (1962) "Woodwind Instruments and their history", Faber, London.

BAK N., DOMLER P. (1987) "The relation between blowing pressure and blowing frequency in clarinet playing", Acustica, 63, 238-241.

BENADE A.H. (1976) "Fundamentals of musical acoustics", Oxford Univ. Press. New York.

BENADE A.H., RICHARDS W.B. (1983) "Oboe normal mode adjustment via reed-staple proportioning", J. Acoust. Soc. Am., 73, 1794-1803.

BOUASSE H. (1929) "Instruments à vent", Delagrave, reedition Blanchard (1986).

BOUTILLON X. (1991) "Analytical Investigation of the flattening effect : The reactive power balance rule", J. Acoust. Soc. Am., 90, 754-763.

BOUTILLON X., GIBIAT V. (1994) "Application expérimentale du bilan de puissance réactive à la mesure de l'anche du saxophone", 3ième Congrés Français d'Acoustique, Toulouse, Suppl. J. Physique III, 4, C5, 665-668.

CASTELLENGO M. (1982) "Sons multiphoniques aux instruments à vent", Rapports IRCAM 34/82, Centre Georges Pompidou, Paris.

COLTMAN J.W. (1968) "Sounding mechanism of the flute and organ pipe", J. Acoust. Soc. Am., 44, 983-992. 
DALMONT J.P., BRUNEAU A.M. (1991) "Acoustic impedance measurement: plane-wave mode and first helical-mode contributions", J. Acoust. Soc. Am., 91, 3026-3033.

DALMONT J.P., GILBERT J. (1993) "Inharmonicity and ease of playing of the saxophone" Proceedings of the Stockholm Music Acoustics Conference, Royal Swedish Academy of Music 79, Stockholm, Sweden.

DALMONT J.P., HERZOG Ph. (1993) "Improved analysis of impedance measurements" Proceedings of the Institute of Acoustics, Southampton, Vol 15, Part 3, 681-688.

DAS P. (1931) "Theory of the clarinet", Indian J. Physics, 6, 225-232.

FLETCHER N.H. (1978) "Mode locking in nonlinearly excited inharmonic musical oscillators", J. Acoust. Soc. Am., 64, 1566-1569.

FLETCHER N.H. (1979) "Excitation mechanisms in woodwind and brass instruments", Acustica, 43, 63-72.

GAZENGEL B. (1994) "Caractérisation objective de la qualité de justesse, de timbre et de facilité d'émission des instruments à vent à anche simple", Thèse de Doctorat de l'Université du Maine, Le Mans, France.

GILBERT J., KERGOMARD J., NGOYA E. (1989) "Calculation of the steady state oscillations of a clarinet using the harmonic balance technique", J. Acoust. Soc. Am., 86, 3541.

Mc GINNIS C.S., GALLAGHER C. (1941) "The mode of Vibration of a Clarinet Reed", J. Acoust. Soc. Am., 12, 529-531.

GRAND N., GILBERT J., LALOE F. (1994) "Influence des nonlinéarités sur les caractéristiques des oscillations des instruments à vent", 3ième Congrés Français d'Acoustique, Toulouse, Suppl. J. Physique III, 4,C5, 577-580

HIRSCHBERG A., VAN DE LAAR R.W.A., MARROU-MAURRIERES J.P., WIJNANDS A.P.J. (1990) "A quasi stationary model of the air flow in the reed channel of single reed woodwind instruments", Acustica, 70, 146-154. 
HIRSCHBERG A., GILBERT J., WIJNANDS A.P.J., HOUTSMA A.J.M. (1991) "Non-linear behaviour of single reed woodwind musical instrument", Nederlands Akoestisch Genootschap, 107, 31-43.

IDOGAWA T., SHIMIZU M., IWAKI M. (1991) "Acoustical behaviour of an oboe and a soprano saxophone artificially blown", in Proceedings of the Symposium on Some Problems on the Theory of Dynamical Systems in Applied Science (World Scientific, Singapore, 1991), Vol. 10.

IDOGAWA T., KOBATA T., KOMURO K., IWAKI M. (1993) "Nonlinear vibrations in the air column of a clarinet artificially blown", J. Acoust. Soc. Am., 93, 540-551.

McINTYRE M.E., SCHUMACHER R.T., WOODHOUSE J. (1983) "On the oscillations of musical instruments", J. Acoust. Soc. Am., 74, 1325-1345.

KEEFE D.H. (1992) "Physical modelling of wind instruments", Computer music journal, $16(4), 57-73$.

KERGOMARD J. (1988) "General equivalent Electric Circuits for Acoustic Horns", J. Audio Eng. Soc., 36, 948-955.

KERGOMARD J., MEYNIAL X. (1988) "Systèmes micro-intervalles pour les instruments de musique à vent avec trous latéraux", J. Acoustique, 1, 255-270.

LYONS D.H. (1981), "Resonance frequencies of the recorder (English flute)", J. Acoust. Soc. Am., 70,1239-1247.

NEDERVEEN C.J. (1967) "Hole calculations for an Oboe", Acustica, 18, 47-57.

NEDERVEEN C.J. (1969) "Acoustical aspects of woodwind instruments", Fritz Knuf pub., Amsterdam.

PLITNIK G.R., STRONG W.J. (1979) "Numerical method for calculating input impedance of the oboe", J. Acoust. Soc. Am., 65, 816-825.

PRATT R.L., BOWSHER J.M. (1979) "The objective assessment of trombone quality", J. Sound Vib., 65, 521-547. 
RESONANS (1984), software for wind-instrument design, developed at LAUM (Le Mans, France) in collaboration with the IRCAM (Paris, France), distributed by CTTM (Le Mans, France).

RUSSEL M.E. (1953) "The Oboe", Dissertation Iowa State Teachers College, Cedar Falls, Iowa.

SANEYOSHI J., TERAMURA H., YOSHIKAWA S. (1987) "Feedback oscillations in reed woodwind and brasswind instruments", Acustica, 62, 194-210.

SCHUMACHER R.T. (1978) "Self sustained oscillations of the clarinet: an integral equation approach", Acustica, 40, 298-309.

SCHUMACHER R.T. (1981) "Ab initio calculations of the oscillation of a clarinet", Acustica, 48, 71-85.

WILSON T.A., BEAVERS G.S. (1974) "Operating modes of the clarinet", J. Acoust. Soc. Am., 56, 653-658.

WORMAN W.E. (1971) "Self-sustained non-linear oscillations of medium amplitude in clarinet-like systems", PhD thesis, Case Western Reserve Univ., Cleveland, Ohio. 


\section{Figures captions}

Figure 1 : Mouthpiece and input of a single reed woodwind instrument.

Figure 2 : Block diagram of a woodwind instrument idealised as a nonlinear element coupled to a passive linear resonator.

Figure 3 : Deviation of the tempered scale expressed in cents for the playing frequency $(*)$ and the first resonance frequency $(+)$. Measured frequencies correspond to the fingerings of the first register (a) and of the second register (b) of a clarinet.

Figure 4 : Deviation of the tempered scale expressed in cents for the playing frequency $(*)$ and the first resonance frequency $(+)$. Measured frequencies correspond to the fingerings of the first register (a) and of the second register (b) of an alto saxophone.

Figure 5 : Measured length correction $\Delta \ell$ (in $\mathrm{mm}$ ) corresponding to the fingering of the first register (a) and the second register (b) of a clarinet.

Figure 6 : Measured length correction $\Delta \ell$ (in $\mathrm{mm}$ ) corresponding to the fingerings of the first register (a) and the second register (b) of an alto saxophone. For the second register (b), two different adjustments of the mouth are represented : case 1 (o) and case $2(*)$.

Figure 7 : Measured deviations of the second resonance frequency due to the register hole opening, case of a clarinet.

Figure 8 : Magnitude of the measured dimensionless input impedance curve for two fingered c'"\# of the clarinet. The usual fingering (a) is using the third resonance. The unusual fingering is the fingering $\mathrm{f}^{\prime} \#$ of the first register with open register hole (b).

Figure 9 : Measured deviations of the second resonance frequency due to the register holes opening, case of an alto saxophone, first register hole $(+)$ and second register hole $(*)$.

Figure 10 : Measured resonance frequencies corresponding to the fingerings of the first register of a clarinet. Figure (a): diagram of tuning deviation for the first resonance frequency $(+)$ and the second resonance frequency $(o)$. Figure (b): diagram of inharmonicity between the two first resonance frequencies. Measured results $(*)$ of unusual fingerings $\left(b, f^{\prime} \#, a^{\prime}\right)$ are presented on figures (a) and (b).

Figure 11 : Measured inharmonicity between the two first resonance frequencies. Comparison between two clarinets of different models, maker A $(+)$ and maker B (o), for the first register fingerings.

Figure 12 : Magnitude of the measured dimensionless input impedance curve for the c"\# fingering of an alto saxophone. 
Figure 13 : Modified neck of an alto saxophone with two branched closed tubes. The tube 1 (respectively 2) is $40 \mathrm{~mm}$ (respectively $165 \mathrm{~mm}$ ) from the input of the neck. The diameter and the maximum height of the two tubes are $12 \mathrm{~mm}$ and $65 \mathrm{~mm}$, respectively.

Figure 14 : Deviations (in cents) on the two first resonance frequencies $\mathrm{f}_{1}$ and $\mathrm{f}_{2}$ (fingerings of the alto saxophone first register) due to the closed tube 2 (height $65 \mathrm{~mm}$ ).

Figure 15 : Deviations (in cents) of the two first resonance frequencies $\mathrm{f}_{1}$ and $\mathrm{f}_{2}$ (fingerings of the alto saxophone first register) due to the closed tube 1 (height $40 \mathrm{~mm}$ ).

Figure 16: Amplitude difference (in $\mathrm{dB}$ ) between the two first harmonics of the c"\# alto saxophone fingering, for 4 different heights of tube 1 .

Figure 17 : Magnitude of the measured dimensionless input impedance curve for the $\mathrm{g}^{\prime}$ fingering of an alto saxophone.

Figure 18 : Notations for the truncated cone with equivalent volume.

Figure 19 : Notations for the two successive cone resonators with equivalent volume.

Figure 20 : Schematic view of the missing part of the cone of the figure 19 (hatched part).

Figure 21 : Inharmonicity of the first two resonance frequencies calculated for different kinds of resonators versus the equivalent length. From the right to the left : truncated single cone without equivalent volume ( $\mathrm{V}=0, \alpha=0, \lambda=0)$, truncated single cone with optimum equivalent volume $\left(\mathrm{V}=\mathrm{V}_{\mathrm{opt}}, \alpha=0, \lambda=0\right)$, double cone with optimum conicity ratio, first case $\left(\mathrm{V}=\mathrm{V}_{\mathrm{opt}}, \alpha\right.$ $\left.=1, \lambda=\lambda_{\mathrm{opt}}=0.03\right)$, Double cone with optimum conicity ratio, second case $\left(\mathrm{V}=\mathrm{V}_{\mathrm{opt}}, \alpha=1 / 3, \lambda\right.$ $=\lambda_{\mathrm{opt}}=0.627$ ).

Figure 22: Theoretical inharmonicity of the first two resonance frequencies for a double cone with optimum volume versus the equivalent length. Upper curve : with optimum conicity ( $\alpha$ $\left.=1 / 3, \lambda=\lambda_{\mathrm{pt}}=0.627\right)$. Lower curve : with non optimum conicity $(\alpha=1 / 3, \lambda=1$. $)$.

Figure 23 : Diameter as a function of the distance to the staple for three different oboes. $5 \mathrm{~mm}$ and $10 \mathrm{~mm}$ respectively have been added to the upper curves to keep the curves separated.

Figure 24 : Deviations to the standard cone. The deviation $\Delta \mathrm{D}$ is defined by the relation $\Delta \mathrm{D}=\mathrm{D}-\mathrm{X} / 40-4 \mathrm{~mm}$. (a) Oboe A, (b) Oboe B, (c) Oboe C.

Figure 25 : Inharmonicity of the first two resonance frequencies for three oboes noted A,B,C, versus the equivalent length defined here as $L=c / 2 f_{1}$, where $f_{1}$ is the first resonance frequency. (a) calculated with the software RESONANS, (b) deduced from impedance measurements. $(*)$, respectively $(+)$ and $(0)$, is corresponding to data of the oboe $\mathrm{A}$, respectively $\mathrm{B}$ and $\mathrm{C}$. 
Figure 26 : Tuning of the first register of three different oboes noted A,B,C. (*), respectively $(+)$ and $(\mathrm{o})$, is corresponding to data of the oboe A, respectively B and $\mathrm{C}$. 